\title{
Generalizing Hartree-Fock theory for nonrelativistic atomic ions to yield a ground-state electron density satisfying cusp and asymptotic conditions
}

\author{
C. Amovilli* \\ Dipartimento di Chimica e Chimica Industriale, Università di Pisa, Via Risorgimento 35, I-56126 Pisa, Italy \\ N. H. March \\ Department of Physics, University of Antwerp, Groenenborgerlaan 171, B-2020 Antwerpen, Belgium \\ J. D. Talman \\ Department of Applied Mathematics, University of Western Ontario, Middlesex College, London, ON, Canada N6A 5B7
}

(Received 12 October 2007; published 7 March 2008)

\begin{abstract}
Möller and Plesset in early work demonstrated quantitatively the high quality of the Hartree-Fock groundstate electron density $\rho_{\mathrm{HF}}(\mathbf{r})$. Therefore, Cordero, March, and Alonso recently proposed, for atoms, a finetuning of $\rho_{\mathrm{HF}}(\mathbf{r})$ to satisfy both the known asymptotic behavior of the density far from the nucleus, as well as the Kato cusp condition at the nuclei. Here we extend the diagonal density $\rho(\mathbf{r})$ proposed by Cordero $e t$ al. to an off-diagonal idempotent one-particle density matrix. The orthonormal orbitals used in this construction are then to be found from the solution of generalized Hartree-Fock equations. We report calculations on the $\mathrm{Be}$ isoelectronic series of atomic ions with $Z$ from $\mathrm{Li}^{-}$to $\mathrm{Ca}^{16+}$.
\end{abstract}

DOI: 10.1103/PhysRevA.77.032503

PACS number(s): 31.15.xr, 31.15.ve, 32.10.-f

\section{INTRODUCTION}

In their pioneering work on perturbation theory built from the Hartree-Fock (HF) method for atoms and molecules as the unperturbed solution, Möller and Plesset [1] demonstrated the high quality of the HF ground-state electron density $\rho_{\mathrm{HF}}(\mathbf{r})$. Specifically, they proved that this density $\rho_{\mathrm{HF}}$ was correct to second order in the difference between the Fock operator and the exact nonrelativistic Hamiltonian.

Motivated by this fact, Cordero et al. [2] have recently proposed, for spherical atoms, a method by which $\rho_{\mathrm{HF}}(r)$ can be "fine-tuned" to satisfy basic physical constraints (a) at the nucleus and (b) asymptotically at large $r$. In particular, Cordero et al. suggested, for atoms,

$$
\rho(\mathbf{r})=\lambda^{3} \rho_{\mathrm{HF}}(\zeta ; \lambda \mathbf{r}),
$$

where $\zeta$ and $\lambda$ are, respectively, an effective nonintegral atomic number and a length scaling factor. $\zeta$ and $\lambda$ are found by satisfying the long range behavior of the density, namely,

$$
\ln \rho \approx-\sqrt{\left(2 I_{\mathrm{NR}}\right)} r
$$

$I_{\mathrm{NR}}$ being the nonrelativistic ionization potential, together with Kato's cusp condition at the nucleus. Cordero et al. proposed to use one piece of experimental data in their "finetuning" of $\rho_{\mathrm{HF}}$, namely, the measured ionization potential. They proposed a way to correct such measurements for modest relativistic effects.

\footnotetext{
*amovilli@dcci.unipi.it

${ }^{\dagger}$ Also at Oxford University, Oxford, England.
}

\section{EQUATIONS FOR ORBITALS YIELDING THE SCALED DENSITY}

In order to find equations leading to the Cordero et al. density in Eq. (1), we first propose an off-diagonal generalization of the Cordero et al. ansatz. Thus we write an idempotent one-particle density matrix (1DM) in terms of orthonormal orbitals $\phi_{j}$ as

$$
\gamma\left(\mathbf{r}_{1} ; \mathbf{r}_{2}\right)=\lambda^{3} \sum_{j}^{\text {occ }} \phi_{j}\left(\zeta ; \lambda \mathbf{r}_{1}\right) \phi_{j}^{*}\left(\zeta ; \lambda \mathbf{r}_{2}\right) .
$$

This is not, of course, claimed to relate to the exact 1DM which is not idempotent, satisfying instead the inequality $\gamma^{2}<\gamma$.

The present proposal is then to find the density $\rho(r)$ in Eq. (1) by solving a generalized HF equation. Assuming an even number of electrons, with $\rho(\mathbf{r})=2 \gamma(\mathbf{r}, \mathbf{r})$, we find the orthonormal orbitals $\phi_{j}$ entering Eq. (3) from the self-consistent set of equations

$$
\begin{aligned}
& {\left[-\frac{1}{2} \nabla^{2}-\frac{\zeta}{r}+\int d \mathbf{r}_{1} \frac{\rho_{\mathrm{HF}}\left(\zeta ; \mathbf{r}_{1}\right)}{\left|\mathbf{r}-\mathbf{r}_{1}\right|}\right] \phi_{j}(\mathbf{r})} \\
& \quad-\int d \mathbf{r}_{1} \frac{\gamma_{\mathrm{HF}}\left(\zeta \mid \mathbf{r} ; \mathbf{r}_{1}\right) \phi_{j}\left(\mathbf{r}_{1}\right)}{\left|\mathbf{r}-\mathbf{r}_{1}\right|}=\epsilon_{j} \phi_{j}(\mathbf{r}) .
\end{aligned}
$$

These equations can be scaled conveniently by putting $\mathbf{r} \rightarrow \lambda \mathbf{r}$ to yield

$$
\begin{aligned}
& {\left[-\frac{1}{2 \lambda^{2}} \nabla^{2}-\frac{\zeta}{\lambda r}+\lambda^{2} \int d \mathbf{r}_{1} \frac{\rho_{\mathrm{HF}}\left(\zeta ; \lambda \mathbf{r}_{1}\right)}{\left|\mathbf{r}-\mathbf{r}_{1}\right|}\right] \phi_{j}(\lambda \mathbf{r})} \\
& -\lambda^{2} \int d \mathbf{r}_{1} \frac{\gamma_{\mathrm{HF}}\left(\zeta \mid \lambda \mathbf{r} ; \lambda \mathbf{r}_{1}\right) \phi_{j}\left(\lambda \mathbf{r}_{1}\right)}{\left|\mathbf{r}-\mathbf{r}_{1}\right|}=\epsilon_{j} \phi_{j}(\lambda \mathbf{r}),
\end{aligned}
$$

where $\gamma_{\mathrm{HF}}$ denotes the $1 \mathrm{DM}$ generalizing $\rho_{\mathrm{HF}}$. Finally, by multiplying both sides by $\lambda^{2}$, we get 


$$
\begin{aligned}
& {\left[-\frac{1}{2} \nabla^{2}-\frac{Z}{r}+\lambda \int d \mathbf{r}_{1} \frac{\rho\left(\mathbf{r}_{1}\right)}{\left|\mathbf{r}-\mathbf{r}_{1}\right|}\right] \psi_{j}(\mathbf{r})} \\
& \quad-\lambda \int d \mathbf{r}_{1} \frac{\gamma\left(\mathbf{r} ; \mathbf{r}_{1}\right) \psi_{j}\left(\mathbf{r}_{1}\right)}{\left|\mathbf{r}-\mathbf{r}_{1}\right|}=\widetilde{\epsilon}_{j} \psi_{j}(\mathbf{r}) .
\end{aligned}
$$

In Eq. (6), we have

$$
Z=\lambda \zeta, \quad \psi_{j}(\mathbf{r})=\sqrt{\lambda^{3}} \phi_{j}(\lambda \mathbf{r}), \quad \tilde{\epsilon}_{j}=\epsilon_{j} \lambda^{2} .
$$

When $\lambda$ is found by solving

$$
-\tilde{\epsilon}_{\mathrm{HOMO}}(\lambda)=I_{\mathrm{NR}}
$$

Equation (6) plays a role of an Euler-Lagrange equation which gives the density in the form of Eq. (1). In this equation, the kinetic and nuclear potential energy terms could arise, in principle, from the functional derivative with respect to orbitals $\psi_{j}$ from

$$
T_{s}+V_{\mathrm{en}}=\int d \mathbf{r}\left[-\nabla_{1}^{2} \gamma\left(\mathbf{r}_{1} ; \mathbf{r}\right)\right]_{\mathbf{r}_{1}=\mathbf{r}}-Z \int d \mathbf{r} \frac{\rho(\mathbf{r})}{r}
$$

while, the electron-electron interaction term, apart for the parameter $\lambda$, comes from the same functional derivative of

$$
G[\gamma]=\frac{1}{2} \int d \mathbf{r}_{1} d \mathbf{r}_{2} \frac{\rho\left(\mathbf{r}_{1}\right) \rho\left(\mathbf{r}_{2}\right)}{\left|\mathbf{r}_{1}-\mathbf{r}_{2}\right|}-\int d \mathbf{r}_{1} d \mathbf{r}_{2} \frac{\gamma\left(\mathbf{r}_{1} ; \mathbf{r}_{2}\right)^{2}}{\left|\mathbf{r}_{1}-\mathbf{r}_{2}\right|}
$$

The eigenvalues $\tilde{\boldsymbol{\epsilon}}_{j}$ are Lagrange multipliers introduced to allow orthogonality constraints to be imposed on the orbitals.

It will be of interest for the future to find, assuming it exists, a functional $f(G)$ satisfying the relation

$$
E \leq T_{s}+V_{\mathrm{en}}+f(G) \leq E_{\mathrm{HF}}
$$

In such case we would have $f^{\prime}(G)=\lambda$.

First of all we must focus attention on the following exact scaling relations

$$
T_{s}+V_{\mathrm{en}}+\lambda G=\lambda^{2} E_{\mathrm{HF}}\left[\frac{Z}{\lambda}\right]
$$

and

$$
\tilde{\epsilon}_{\mathrm{HOMO}}=\lambda^{2} \epsilon_{\mathrm{HF}}^{\mathrm{HOMO}}\left[\frac{Z}{\lambda}\right] .
$$

The two functions $E_{\mathrm{HF}}(x)$ and $\epsilon_{\mathrm{HF}}^{\mathrm{HOMO}}(x)$, to be determined for an isoelectronic series, provide all the required information. If we write

$$
E(\lambda)=T_{s}+V_{\text {en }}+\lambda G+\Delta(\lambda)
$$

with $\Delta(\lambda)$ at the present unknown, $E$ will have a minimum for the optimal value of $\lambda, \lambda$ being the unique variational parameter in scaling $\rho$. Making use of Eq. (12), we can write

$$
2 \lambda_{0} E_{\mathrm{HF}}\left[\frac{Z}{\lambda_{0}}\right]-Z E_{\mathrm{HF}}^{\prime}\left[\frac{Z}{\lambda_{0}}\right]+\Delta^{\prime}\left(\lambda_{0}\right)=0
$$

with $\lambda_{0}$ minimizing $E(\lambda)$. Equation (15) is the analog of Eq. (8), namely,

$$
-\lambda_{0}^{2} \epsilon_{\mathrm{HF}}^{\mathrm{HOMO}}\left[\frac{Z}{\lambda_{0}}\right]=I_{\mathrm{NR}}(Z) .
$$

We can relate Eqs. (15) and (16) to find a reliable form for the function $\Delta(\lambda)$. Because $\lambda_{0} \approx 1$ it is convenient to expand in terms of $\delta=1-\lambda$, truncating at the first order in $\delta$. To this order we have, from Eq. (16),

$$
\lambda_{0}=1-\frac{I_{\mathrm{NR}}+\epsilon}{2 \epsilon-Z \epsilon^{\prime}},
$$

where, for simplicity, $\epsilon=\epsilon_{\mathrm{HF}}^{\mathrm{HOMO}}(Z)$. To get the same $\lambda_{0}$ from Eq. (15), $\Delta$ must satisfy the following conditions:

$$
\Delta^{\prime}(1)=k\left(I_{\mathrm{NR}}+\epsilon\right)-2 E_{\mathrm{HF}}(Z)+Z E_{\mathrm{HF}}^{\prime}(Z)
$$

and

$$
\Delta^{\prime \prime}(1)=k\left(2 \epsilon-Z \epsilon^{\prime}\right)-2 E_{\mathrm{HF}}(Z)+2 Z E_{\mathrm{HF}}^{\prime}(Z)-Z^{2} E_{\mathrm{HF}}^{\prime \prime}(Z),
$$

where $k$ is a common constant. The function $\Delta(\lambda)$ can now be reconstructed to the second order of a Taylor expansion in $\delta$. Recalling that $\Delta(1)=0$, we have

$$
\begin{aligned}
\Delta(\lambda) & =-\int_{\lambda}^{1}\left[\Delta^{\prime}(1)+\Delta^{\prime \prime}(1)(\eta-1)\right] d \eta \\
& =\frac{1}{2} \Delta^{\prime \prime}(1)-\Delta^{\prime}(1)+\left[\Delta^{\prime}(1)-\Delta^{\prime \prime}(1)\right] \lambda+\frac{1}{2} \Delta^{\prime \prime}(1) \lambda^{2}
\end{aligned}
$$

because we must require $E(1)=E_{\mathrm{HF}}(Z)$ from Eq. (14). To complete the description of $\Delta(\lambda)$, one needs a value for $k$ defined above. A reasonable choice should be that which guarantees Eq. (11) for $\lambda$ ranging in the interval between $\lambda_{0}$ and 1 for a wide interval of $Z$ values. $k$ can be dependent on $Z$. Such a quantity $\Delta(\lambda)$ in this context plays the role of a penalty function, forcing $\lambda$ to be $\lambda_{0}$ in such a way that orthonormal orbitals $\psi_{j}$ can be determined via Eq. (6). $\Delta(\lambda)$ is a positive function which could include corrections for the incorrect counting of electron-electron interactions in $\lambda G$ $[(1-\lambda) N(N-1) / 2$ are missing $]$ and for correlation kinetic energy (here we have only $T_{s}$ ).

For the future, it will be of interest to investigate whether it is possible to construct an energy functional in the form

$$
E[\gamma, \lambda]=T_{s}+V_{\mathrm{en}}+\lambda G-\int_{\lambda}^{1} h[\gamma](\eta) d \eta
$$

with a more general, and presently unknown, functional $h[\gamma]$ instead of the penalty function $\Delta(\lambda)$ introduced above. For 
TABLE I. Accurate Hartree-Fock energy contributions $(\lambda=1)$, calculated in this work, for the Be isoelectronic series of atomic ions and accurate nonrelativistic ionization potentials and energies taken from literature [4-6]. All values are in atomic units.

\begin{tabular}{rrrrrrrr}
\hline \hline$Z$ & \multicolumn{1}{c}{$T_{s}(1)$} & $V_{\mathrm{en}}(1)$ & $G(1)$ & $E_{\mathrm{HF}}$ & $\epsilon_{\mathrm{HF}}^{\mathrm{HOMO}}$ & $I_{\mathrm{NR}}$ & $E_{\text {lit }}$ \\
\hline 3 & 7.42823 & -17.61003 & 2.75357 & -7.42823 & -0.01454 & 0.0228 & -7.50040 \\
4 & 14.57301 & -33.63515 & 4.48912 & -14.57301 & -0.30927 & 0.3426 & -14.66735 \\
5 & 24.23761 & -54.59330 & 6.11811 & -24.23757 & -0.87381 & 0.9245 & -24.34887 \\
6 & 36.40847 & -80.53612 & 7.71915 & -36.40848 & -1.69404 & 1.7599 & -36.53482 \\
7 & 51.08228 & -111.47320 & 9.30862 & -51.08229 & -2.76673 & 2.8472 & -51.22269 \\
8 & 68.25758 & -147.40736 & 10.89210 & -68.25767 & -4.09071 & 4.1858 & -68.41152 \\
9 & 87.93388 & -188.34000 & 12.47213 & -87.93398 & -5.66546 & 5.7758 & -88.10090 \\
10 & 110.11063 & -234.27147 & 14.04994 & -110.11089 & -7.49070 & 7.6172 & -110.29063 \\
11 & 134.78769 & -285.20217 & 15.62626 & -134.78820 & -9.56628 & 9.7104 & -134.9823 \\
12 & 161.96496 & -341.13229 & 17.20155 & -161.96578 & -11.89209 & 12.0557 & -162.1723 \\
13 & 191.64228 & -402.06190 & 18.77606 & -191.64355 & -14.46807 & 14.6536 & -191.8625 \\
14 & 223.81978 & -467.99125 & 20.35003 & -223.82143 & -17.29418 & 17.5045 & -224.0527 \\
15 & 258.49688 & -538.91977 & 21.92350 & -258.49938 & -20.37039 & 20.6087 & -258.74167 \\
16 & 295.67379 & -614.84777 & 23.49663 & -295.67735 & -23.69667 & 23.9678 & -295.9329 \\
17 & 335.35039 & -695.77517 & 25.06948 & -335.35530 & -27.27302 & 27.5813 & -335.6230 \\
18 & 377.52654 & -781.70183 & 26.64208 & -377.53320 & -31.09942 & 31.4505 & -377.8129 \\
19 & 422.20213 & -872.62765 & 28.21449 & -422.21102 & -35.17585 & 35.5758 & -422.5027 \\
20 & 469.37711 & -968.55256 & 29.78673 & -469.38871 & -39.50231 & 39.9596 & -469.69419 \\
\hline \hline
\end{tabular}

the present, however, we illustrate the above theory based on a nonlocal potential on some spherical atomic ions.

\section{ILLUSTRATIVE EXAMPLES: BE ISOELECTRONIC SERIES OF ATOMIC IONS}

To test the theory presented here, we carried out calculations on the Be isoelectronic series of atomic ions with $Z$ from $\mathrm{Li}^{-}$to $\mathrm{Ca}^{16+}$. Hartree-Fock data have been calculated using a basis set of 30 even tempered Gaussian functions [3] (exponents $\alpha \beta^{j}$ ) with $\alpha=0.00015, \beta=2$, and $0 \leq j \leq 29$.

In Table I, Hartree-Fock data are collected together with ionization potentials and accurate correlated energies taken from the literature [4-6]. The virial theorem $\left[T_{s}(1)=-E_{\mathrm{HF}}\right]$ and the Hellmann-Feynman theorem $\left[d E_{\mathrm{HF}} / d Z=V_{\mathrm{en}}(1) / Z\right]$ are satisfied to good accuracy. However, we can bring the HF data in column 5 of Table I into contact with the work by Davidson et al. [7] which solves the HF equations numerically. The differences between the two sets of data are a small fraction of the correlation energy which is a focus of the present study. The values of the total energy reported in the last column of Table I taken from Refs. [4-6] are very close to those reported by Chakravorty et al. [8], the differences not changing any of the present conclusions.

Apart for $\mathrm{Li}^{-}$, which shows a nuclear charge close to the critical value for which $I_{\mathrm{NR}}$ tends to zero, correlation energies are well reproduced by the choice

$$
k(Z)=-5 Z+112+\frac{345}{Z} .
$$

This expression is reminiscent of the $1 / Z$ expansion (see Refs. [8] and [9]). However, another quantity $\epsilon$ appearing in Eqs. (18) and (19) involving $k$ is also a function of $1 / Z$. Therefore, the relation of $k$ to the $1 / Z$ expansion is somewhat indirect and we shall not pursue the matter further.

In order of appearance, Figs. 1 and 2 below show (i) $\lambda_{0}(Z)$ obtained from Eq. (17), which coincides with that obtained from Eq. (8), (ii) the comparison between our recalculated correlation energies and the corresponding values from the literature, and (iii) the plot of $E(\lambda)-E_{\mathrm{HF}}$ for some selected Be-like ions. Finally, Figs. 3-5 show plots of radial densities from diffusion quantum Monte Carlo (DMC), HF, and this work for $\mathrm{Li}^{-}, \mathrm{Be}$, and $\mathrm{Ne}^{6+}$. DMC results refer to calculations performed starting from a guiding function obtained at a variational quantum Monte Carlo (VMC) level with a functional form for the wave function which goes beyond the HF level (standard Slater-Jastrow types). The $\mathrm{Li}^{-}$ plot shows clearly the pathological, but expected, behavior of $\mathrm{Li}^{-}$in this context.

\section{SUMMARY AND FUTURE DIRECTIONS}

Cordero et al. [2] proposed to use a fractional nuclear charge near to the "real" atomic number, for neutral atoms such as, for example, $\mathrm{Mg}$ and $\mathrm{Ar}$, plus a measured ionization potential corrected for relativity, to correct $\rho_{\mathrm{HF}}$ at (i) large distances from the nucleus and (ii) short distances, by satisfying the Kato cusp condition at the nucleus.

The achievement of the present study is to effect an offdiagonal generalization of the Cordero et al. ground-state electron density (1) in the form of the idempotent 1DM set out in Eq. (3). Then the orthonormal orbitals $\phi_{j}$ entering that equation are shown to satisfy generalized HF equations, 

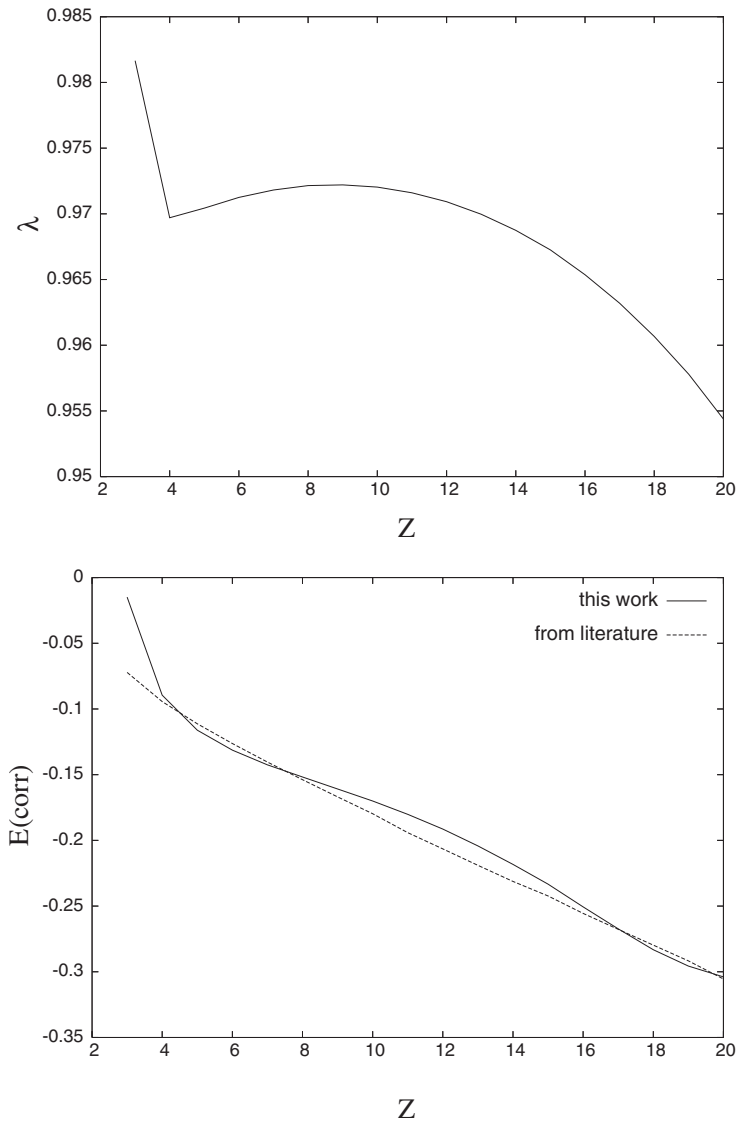

FIG. 1. Optimal $\lambda$ (upper plot), obtained by means of Eq. (17) and comparison of computed correlation energies with corresponding literature data (lower plot) for the Be isoelectronic series of atomic ions considered in this work $(3 \leq Z \leq 20)$. Data are in atomic units.

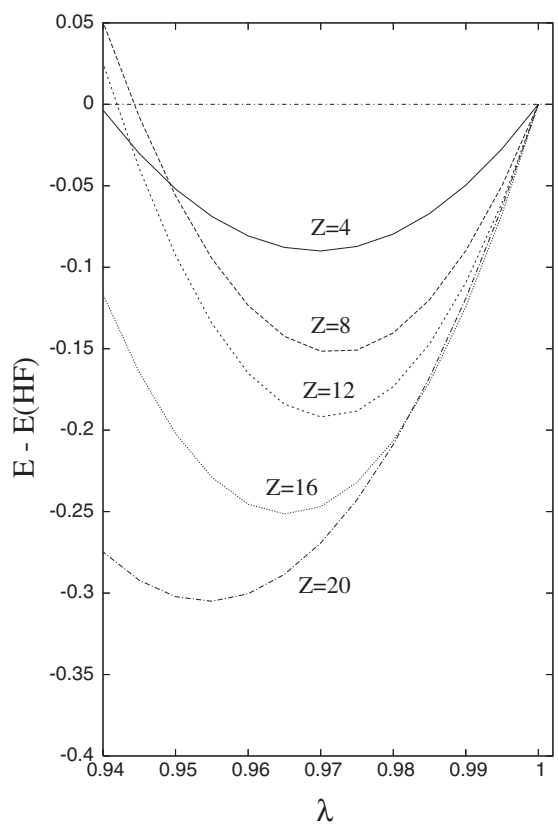

FIG. 2. Plot of the energy relative to the Hartree-Fock energy as a function of the length scale parameter $\lambda$ for different Be-like atomic ions. Energies are in Hartree.

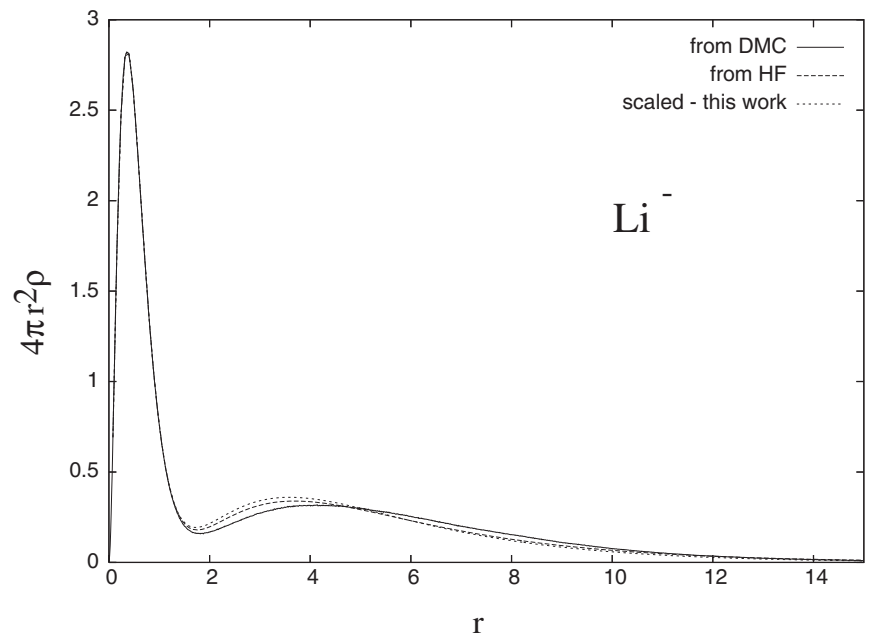

FIG. 3. Plot of the radial density $4 \pi r^{2} \rho(r)$ as a function of the distance from the nucleus for $\mathrm{Li}^{-}$ion. Data are in atomic units.

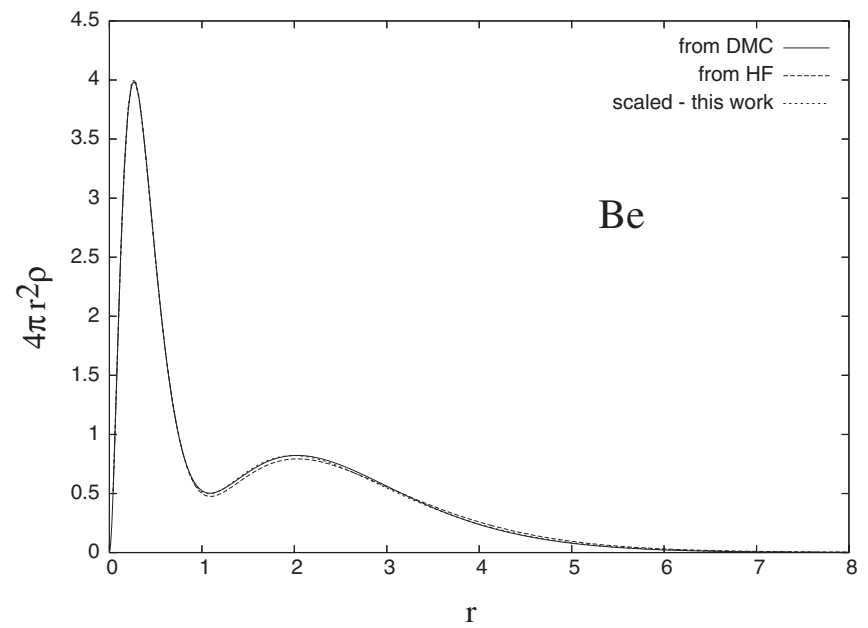

FIG. 4. Plot of the radial density $4 \pi r^{2} \rho(r)$ as a function of the distance from the nucleus for Be. Data are in atomic units.

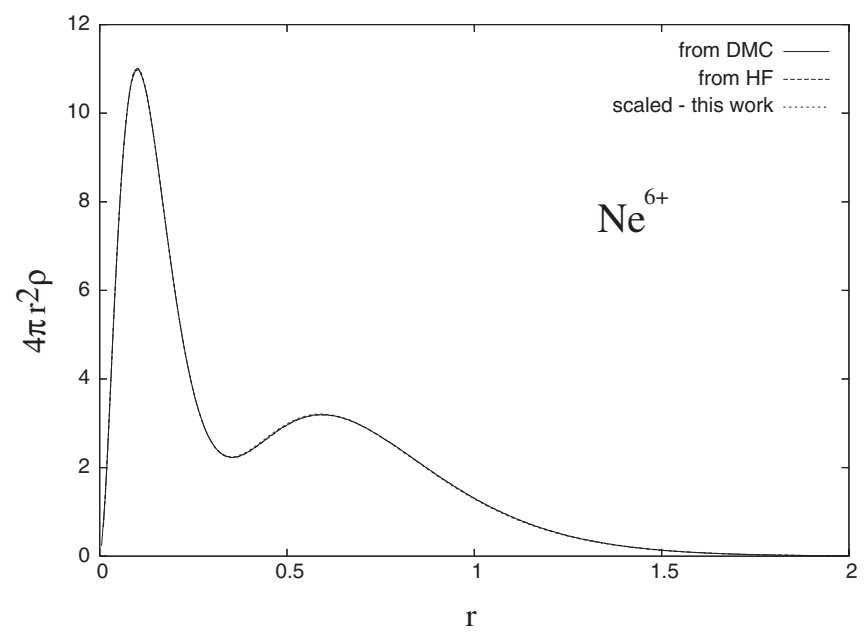

FIG. 5. Plot of the radial density $4 \pi r^{2} \rho(r)$ as a function of the distance from the nucleus for $\mathrm{Ne}^{6+}$ ion. Data are in atomic units. 
based fundamentally on a nonlocal potential. Encouraging numerical results are presented for the Be isoelectronic series of atomic ions.

As to future work, we have conjectured the existence of a functional $f(G)$ entering the inequality (11), where $G$ is given in Eq. (10). This would formally complete the present proposal. However, as shown above for Be-like atomic ions, numerical implementation of the present scheme based on generalized HF equations with a nonlocal potential is already entirely practicable.
[1] C. Möller and M. S. Plesset, Phys. Rev. 46, 618 (1934).

[2] N. A. Cordero, N. H. March, and J. A. Alonso, Phys. Rev. A 75, 052502 (2007).

[3] K. Ruedenberg, R. C. Raffenetti, and R. D. Bardo, Energy, Structure and Reactivity, Proceedings of the 1972 Boulder Seminar Research Conference on Theoretical Chemistry, edited by D. W. Smith (Wiley, New York, 1973), p. 164.

[4] J. S. Sims, S. A. Hagstrom, D. Munch, and C. F. Bunge, Phys. Rev. A 13, 560 (1976).
[5] K. T. Chung, X. W. Zhu, and Z. W. Wang, Phys. Rev. A 47, 1740 (1993).

[6] J. Komasa, J. Rychlewski, and K. Jankowski, Phys. Rev. A 65, 042507 (2002).

[7] E. R. Davidson, S. A. Hagstrom, S. J. Chakravorty, V. M. Umar, and C. F. Fischer, Phys. Rev. A 44, 7071 (1991).

[8] S. J. Chakravorty, S. R. Gwaltney, E. R. Davidson, F. A. Parpia, and C. F. Fischer, Phys. Rev. A 47, 3649 (1993).

[9] N. H. March and R. J. White, J. Phys. B 5, 466 (1972). 